\title{
Recent development in thermoelastic stress analysis
}

\author{
by S. Jecic and S. Goja
}

Faculty Of Mechanical Engineering and Naval Architecture, University of Zagreb

Ivana Lucica 5, 10000 Zagreb, Croatia

\begin{abstract}
Thermoelastic stress analysis (TSA) is one of the methods in experimental mechanics used for qualitative and quantitative estimation of the stress state on the surface of cyclically loaded structures which can be either models or actual structures in their operating environment. In this paper critical remarks are given on two different theoretical approaches to derive the main TSA equation of isotropic materials. The main TSA equation of generally anisotropic and orthotropic materials is also shown. Furthermore, the influence of temperature on thermomechanical features of the materials is investigated. By comparing several construction and model materials it is shown that makrolon is the most suitable material for model making in the thermoelastic stress analysis.
\end{abstract}

\section{Introduction}

Thermography is based on measuring the intensity of the infrared radiation emitted by an elastic structure under cyclic loading. Provided that loading parameters are correctly selected, an isentropic change of state is induced within the structure with mechanical energy transforming into thermal and vice versa. In consequence, that the minor temperature changes with values around $10^{-3} \mathrm{~K}$ can be measured on the surface of the particular structure with specially sensitive thermographic cameras. They can subsequently be correlated to the stress state. The equipment for stress pattern analysis by thermal emission (SPATE) consists of an infrared detector, a lock-in analyser, and a computer.

\section{Theoretical basis}

The state of stress on the surface of the analysed model or machine part and the SPATE system output signal is correlated by the main thermoelastic stress analysis equation. As two different approaches to isotropic materials are prevalent in literature so far, there are two main TSA equations which are mostly used without any commentary or instruction on how to interpret them correctly. By using the first approach [1-3] the next expression was derived:

$$
\left(\sigma_{11}+\sigma_{22}\right)=A \Sigma
$$

which correlates the first stress invariant on the surface to the output signal, but only provided that pure cycling loading is applied $\left(\sigma_{m}=0\right)$. The second approach is not limited to pure cycling loading, and the derived expression correlates the changes in the first stress invariant and output thermoelastic signal [4],

$$
\left(\Delta \sigma_{11}+\Delta \sigma_{22}\right)=A \Sigma
$$

Due to the growing usage of composites, i.e. generally anisotropic materials, a need has recently developed to analyse the state of stress of those materials. Experimental methods are adequate in the stress and strain analysis of such materials, since it is difficult to describe their geometrical and mechanical features. Simultaneously with the development of measuring systems there have thus been expressions derived in an analytical way, which correlates the state of stress to small temperature changes on the surface of a cyclically loaded model. For an orthotropic solid this expression is

$$
\Delta T=-\frac{T_{o}}{\rho \mathcal{C}_{\varepsilon}}\left(\alpha_{11} \Delta \sigma_{11}+\alpha_{22} \Delta \sigma_{22}\right)
$$


The fraction in front of the bracket in the second part of the expression represents the thermoelastic constant $K$

$$
K=\frac{T_{o}}{\rho c_{s}}
$$

Eventually the main thermoelastic equation for orthotropic materials is derived:

$$
\left(\alpha_{11} \Delta \sigma_{11}+\alpha_{22} \Delta \sigma_{22}\right)=A \Sigma
$$

However, this expression must be interpreted correctly, since in this case the temperature change $\Delta T$ (3) (i.e. the output signal (5) ) is not proportional to the change of the first stress invariant (which is the case of isotropic materials) but it is a linear combination of principal stresses along the material orthotropic axes on the surface of a cyclically loaded model. Thereby $\alpha_{11}$ and $\alpha_{22}$ represent thermal expansion coefficients in directions along the material orthotropic axes.

One of the problems is a possible change of either thermal or mechanical material constants, which are both part of the thermoelastic constant. On one side there is a tendency towards an ever higher constant $K$, since its higher values means a stronger thermoelastic effect, i.e. the temperature changes are in that case stronger due to the thermoelastic effect $((3)$ and (4)). The diagram shown in Figure 1 results in the conclusion that model materials Araldite $B$ and Makrolon are characterised by a stronger thermoelastic effect than steel and aluminium which are among the most often used materials in engineering. On the other hand, the thermoelastic effect may cause a change of the above mentioned material constants. The thermoelastic constant for isotropic materials is:

$$
K=\frac{\alpha T_{o}}{\frac{3 T_{o} \alpha^{2} E}{1-2 \nu}+\rho c_{\varepsilon}}
$$

On the basis of the above expression the diagrams in figures 2,3 and 4 show the correlation between the constant $K$ and a possible feature changes in some materials induced by temperature changes. The diagrams show that thermomechanical features of Araldite $B$ and Makrolon are sensitive to temperature changes due to the thermoelastic effect.

\section{Conclusion}

As it can be deducted from the above diagrams, these changes are only slight and can therefore be neglected in the praxis $[2,5]$. The same can be concluded for orthotropic materials, i.e. for anisotropic materials in general $[6,7]$, although this correlation should be investigated in more detail in the course of future research. Further problems to be solved are: calibration of the measuring system, stress separations, sensitivity of the thermographic camera, automation of the procedure, etc.

\section{REFERENCES}

[1] JECIC (S.) and KRPANEC (G.) - Thermoelasticity in Experimental Stress Analysis. Proceedings of Faculty of Mechanical Engineering and Naval Architecture XVI, Faculty of Mechanical Engineering and Naval Architecture Zagreb, 1992, p. 11-18.

[2] NOWACKI (W.) - Thermoelasticity. Polish Scientific Publisher, Warszawa, 1986.

[3] JECIC (S.) and GOMERCIC (M.) and KRPANEC (G.) - Anwendung von Makrolon in der experimentellen Spannungsanalyse. OIAZ, 141, 4/1996, p. 156-160.

[4] GOJA (S.). - Thermoelastic Stress Analysis in Orthotropic Parts of Mechanical Constructions. Master Thesis, Faculty of Mechanical Engineering and Naval Architecture, University of Zagreb, 1996. 
http://dx.doi.org/10.21611/qirt.1998.016

[5] RAUCH (B. J.) and ROWLANDS (R. E.) - Thermoelastic Stress Analysis. Handbook on Experimental Mechanics, 1987, chapter 14, p. 581-599.

[6] FICKER (E.) - Der Einfluss des Modellmaterials in der Spannungsoptik. Dissertation, München, 1971.

[7] WOLF (H.) - Spannungsoptik, Band 1 - Grundlagen. Springer-Verlag, Berlin, 1976.

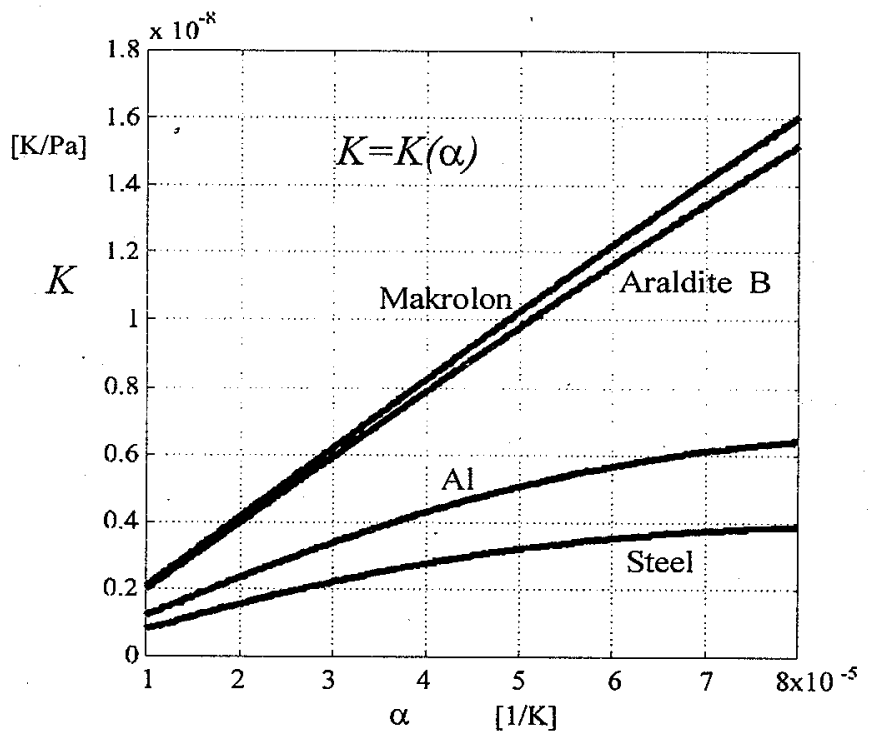

Fig. 1. Thermoelastic effect for various materials

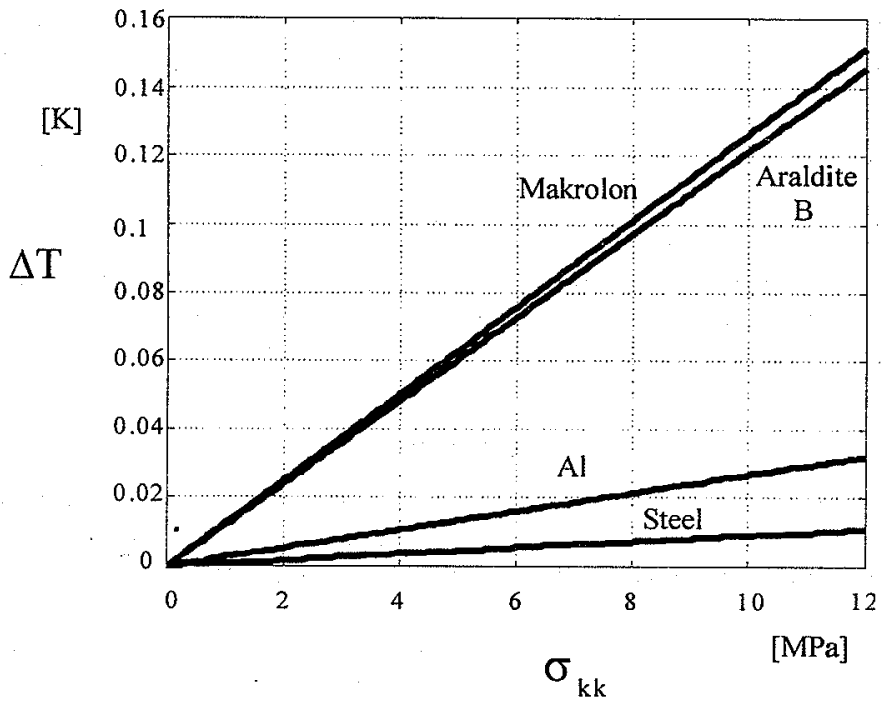

Fig. 2. Dependence of the thermoelastic constant $K$ on the thermal expansion coefficient $\alpha$ 
http://dx.doi.org/10.21611/qirt.1998.016

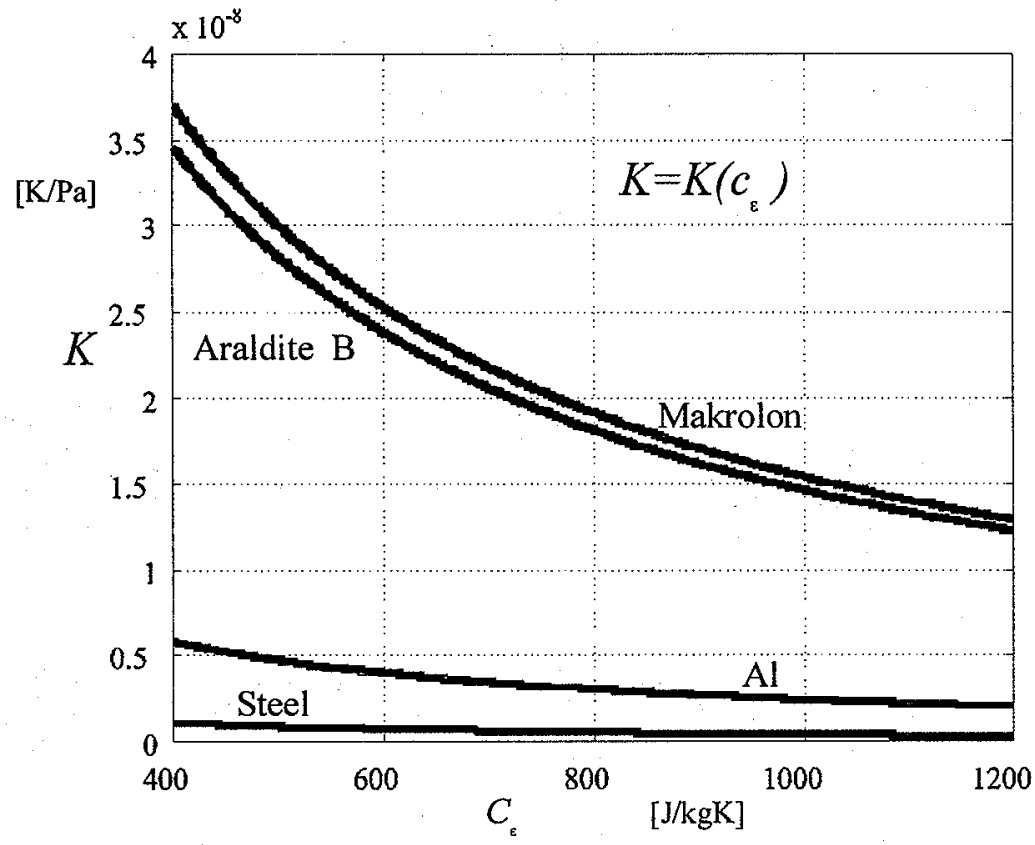

Fig. 3. Dependence of the thermoelastic constant $K$ on the specific heat $c_{\varepsilon}$

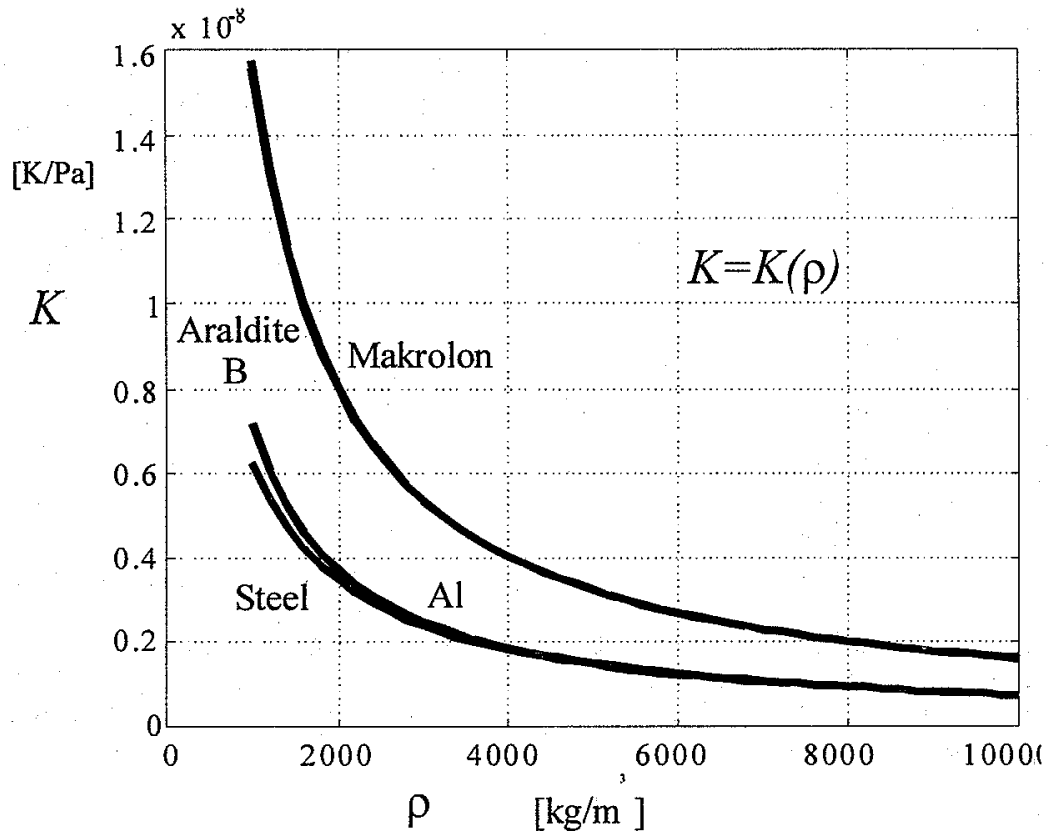

Fig. 4. Dependence of the thermoelastic constant $K$ on the density $\rho$ 